\title{
Baryon resonances from a novel fat-link fermion action
}

\author{
W. Melnitchouk ${ }^{a}, b$, S. Bilson-Thompson ${ }^{a}$, F. D. R. Bonnet ${ }^{a}$, P. D. Coddington ${ }^{a}$, D. B. Leinweber ${ }^{a}$, \\ A. G. Williams ${ }^{a}$, J. M. Zanotti ${ }^{a}$, J. B. Zhang ${ }^{a}$ and F. X. Lee Le $^{b, c}$

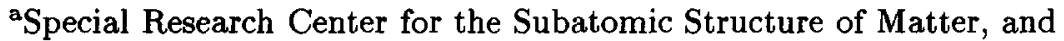 \\ Department of Physics and Mathematical Physics, Adelaide University, 5005, Australia \\ ${ }^{b}$ Jefferson Lab, 12000 Jefferson Avenue, Newport News, VA 23606, U.S.A. \\ ${ }^{c}$ Center for Nuclear Studies, Department of Physics, \\ The George Washington University, Washington, D.C. 20052, U.S.A.
}

We present first results for masses of positive and negative parity excited baryons in lattice QCD using an $\mathcal{O}\left(a^{2}\right)$ improved gluon action and a Fat Link Irrelevant Clover (FLIC) fermion action in which only the irrelevant operators are constructed with fat links. The results are in agreement with earlier calculations of $N^{*}$ resonances using improved actions and exhibit a clear mass splitting between the nucleon and its chiral partner, even for the Wilson fermion action. The results also indicate a splitting between the lowest $J^{P}=\frac{1}{2}^{-}$states for the two standard nucleon interpolating fields.

\section{INTRODUCTION}

Understanding the dynamics responsible for baryon excitations provides valuable insight into the forces which confine quarks inside baryons and into the nature of QCD in the nonperturbative regime. One of the long-standing puzzles in spectroscopy has been the low mass of the first positive parity excitation of the nucleon (the $J^{P}=\frac{1}{2}^{+} N^{*}(1440)$ Roper resonance) compared with the lowest lying odd parity excitation. Another challenge for spectroscopy is presented by the $\Lambda^{1 / 2-}(1405)$, whose anomalously small mass has been interpreted as indicating strong coupled channel effects involving $\Sigma \pi, \bar{K} N, \cdots[1]$, and a weak overlap with a three valence constituent quark state.

In this paper we present the first results of excited octet mass simulations using an $\mathcal{O}\left(a^{2}\right)$ improved gluon action and an improved Fat Link Irrelevant Clover (FLIC) [2] quark action in which only the irrelevant operators are constructed using fat links. Configurations are generated on the new Orion computer cluster dedicated to lattice gauge theories at the CSSM at Adelaide University. After reviewing in Section 2 the main elements of lattice calculations of excited hadron masses, we describe in Section 3 various features of interpolating fields used in this analysis. In Section 4 we present results for $J^{P}=\frac{1}{2}^{ \pm}$nucleons and hyperons. Finally, in Section 5 we make concluding remarks and discuss some future extensions of this work.

\section{BARYONS ON THE LATTICE}

The history of excited baryons on the lattice is quite brief, although recently there has been growing interest in finding new techniques to isolate excited baryons, motivated partly by the experimental $N^{*}$ program at Jefferson Lab. Previous work on excited baryons on the lattice can be found in Refs. [3-7].

Following standard notation, we define a twopoint correlation function for a baryon $B$ as:

$G_{B}(t, \vec{p}) \equiv \sum_{\vec{x}} e^{-i \vec{p} \cdot \vec{x}}\left\langle 0\left|T \chi_{B}(x) \bar{\chi}_{B}(0)\right| 0\right\rangle$,

where $\chi_{B}$ is a baryon interpolating field transforming positively under parity operation, and we have suppressed Dirac indices. The choice of interpolating field $\chi_{B}$ is discussed in Section III below. For large Euclidean time, the correlation function can be written as a sum of the lowest en- 
ergy positive and negative parity contributions:

$$
\begin{aligned}
G_{B}(t, \vec{p}) & \approx \lambda_{B^{+}}^{2} \frac{\left(\gamma \cdot p+M_{B^{+}}\right)}{2 E_{B^{+}}} e^{-E_{B^{+}} t} \\
& +\lambda_{B^{-}}^{2} \frac{\left(\gamma \cdot p-M_{B^{-}}\right)}{2 E_{B^{-}}} e^{-E_{B^{-}} t}
\end{aligned}
$$

where a fixed boundary condition in the time direction is used to remove backward propagating states, and where the overlap of the field $\chi_{B}$ with positive or negative parity states $\left|B^{ \pm}\right\rangle$is parameterized by a coupling strength $\lambda_{B^{ \pm}}$, with $E_{B^{ \pm}}=\sqrt{M_{B^{ \pm}}^{2}+\vec{p}^{2}}$ the energy. The energies of the positive and negative parity states are obtained by taking the trace of $G_{B}$ with the operator $\Gamma_{ \pm}$, where

$$
\Gamma_{ \pm}=\frac{1}{2}\left(1 \pm \frac{M_{B^{ \pm}}}{E_{B^{ \pm}}} \gamma_{4}\right)
$$

For $\vec{p}=0, E_{B^{ \pm}}=M_{B^{ \pm}}$and the operator $\Gamma_{ \pm}$ projects out the mass, $M_{B^{ \pm}}$, of the baryon $B^{ \pm}$. In this case, positive parity states propagate in the 1, 1 and 2, 2 elements of the Dirac matrix of Eq. (2), while negative parity states propagate in the 3,3 and 4,4 elements.

\section{INTERPOLATING FIELDS}

In this analysis we consider two types of interpolating fields which have been used in the literature. The notation adopted follows that of Leinweber et al. [8]. For the positive parity proton we use as interpolating fields:

$\chi_{1}^{p+}(x)=\epsilon_{a b c}\left(u_{a}^{T}(x) C \gamma_{5} d_{b}(x)\right) u_{c}(x)$,

and

$\chi_{2}^{p+}(x)=\epsilon_{a b c}\left(u_{a}^{T}(x) C d_{b}(x)\right) \gamma_{5} u_{c}(x)$,

where the fields $u, d$ are evaluated at Euclidean space-time point $x, C$ is the charge conjugation matrix, $a, b$ and $c$ are color labels, and the superscript $T$ denotes the transpose. As pointed out by Leinweber [3], because of the Dirac structure of the "diquark" in the parentheses in Eq. (4), the field $\chi_{1}^{p+}$ involves both products of upper $\times$ upper $\times$ upper and lower $\times$ lower $\times$ upper components of spinors for positive parity baryons, so that in the nonrelativistic limit $\chi_{1}^{p+}=\mathcal{O}(1)$. Furthermore, since the "diquark" couples to a total spin 0 , one expects an attractive force between the two quarks, and hence a lower energy state than for a state in which two quarks do not couple to spin 0 .

The $\chi_{2}^{p+}$ interpolating field, on the other hand, is known to have little overlap with the ground state $[3,9]$. Inspection of the structure of the Dirac matrices in Eq. (5) reveals that it involves products of upper $\times$ lower $\times$ lower components only for positive parity baryons, so that $\chi_{2}^{p+}=$ $\mathcal{O}\left(p^{2} / E^{2}\right)$ vanishes in the nonrelativistic limit. As a result of the mixing, the "diquark" term contains a factor $\vec{\sigma} \cdot \vec{p}$, meaning that the quarks no longer couple to spin 0 , but are in a relative $L=1$ state. One expects therefore that two-point correlation functions constructed from the interpolating field $\chi_{2}^{p+}$ are dominated by larger mass states than those arising from $\chi_{1}^{p+}$.

Interpolating fields for a negative parity proton can be constructed by multiplying the positive parity fields by $\gamma_{5}, \chi^{B-} \equiv \gamma_{5} \chi^{B+}$, which reverses the role of the terms in Eq. (2). While the masses of negative parity baryons are obtained directly from the (positive parity) interpolating fields in (4) and (5) by using the parity projectors $\Gamma_{ \pm}$, it is instructive nevertheless to examine the general properties of the negative parity interpolating fields. In contrast to the positive parity case, both the interpolating fields $\chi_{1}^{p-}$ and $\chi_{2}^{p-}$ mix upper and lower components, and consequently both $\chi_{1}^{p-}$ and $\chi_{2}^{p-}$ are $\mathcal{O}(p / E)$. Physically, two nearby $J^{P}=\frac{1}{2}^{-}$states are observed in the nucleon spectrum. In simple quark models, the splitting of these two orthogonal states is largely attributed to the extent to which scalar diquark configurations compose the wavefunction [4]. It is reasonable to expect $\chi_{1}^{p-}$ to have better overlap with scalar diquark dominated states, and thus provide a lower effective mass in the large Euclidean time regime explored in lattice simulations. If the effective mass associated with the $\chi_{2}^{p}$ correlator is larger, then this would be evidence of significant overlap of $\chi_{2}^{p-}$ with the higher lying $N^{\frac{1}{2}-}$ states. In this event, further analysis directed at resolving these two states is warranted. 


\section{RESULTS}

In this paper we report results of calculations of octet excited baryon masses performed on a $16^{3} \times 32$ lattice at $\beta=4.60$ with a lattice spacing of $a=0.125(2) \mathrm{fm}$. The analysis is based on a preliminary sample of 50 configurations generated on the new Orion computer cluster at the CSSM, Adelaide. For the gauge fields, a meanfield improved plaquette plus rectangle action is used, while for the quark fields, the FLIC [2] action is implemented. In the present analysis we have imposed fixed boundary conditions in the time direction $\left(U_{t}(\vec{x}, n t)=0 \forall \vec{x}\right)$, and periodic boundary conditions in spatial directions. Although the simulations were performed with both $n=4$ and 12 fattening sweeps, the improved gauge fields were found to be smooth after only 4 sweeps. Since the results with $n=4$ sweeps exhibit slightly better scaling than those with $n=12$ [2], we shall focus on the results with 4 smearing sweeps. The 12 sweep results lead to the same conclusions as presented in the following. Further details of the simulations are given in Ref.[2].

In Fig. 1 we show the $N$ and $N^{*}\left(\frac{1}{2}^{-}\right)$masses as a function of the squared pseudoscalar meson mass, $m_{\pi}^{2}$. The results of the new simulations are indicated by the filled symbols (filled circles are FLIC; filled diamonds are Wilson). For comparison, we also show results from earlier simulations with domain wall fermions (DWF) [6] (open triangles), and a nonperturbatively (NP) improved clover action at $\beta=6.2[7]$. The scatter of the different NP improved results is due to different source smearing and volume effects: the open squares are obtained by using fuzzed sources and local sinks, the open circles use Jacobi smearing at both the source and sink, while the open diamonds, which extend to smaller quark masses, are obtained from a larger lattice $\left(32^{3} \times 64\right)$ using Jacobi smearing. The empirical masses of the nucleon and the lowest $\frac{1}{2}^{-}$excitation are indicated by the asterisks along the ordinate. There is excellent agreement between the different improved actions for the nucleon mass, in particular between the FLIC, NP improved clover [7] and DWF [6] results. On the other hand, the Wilson

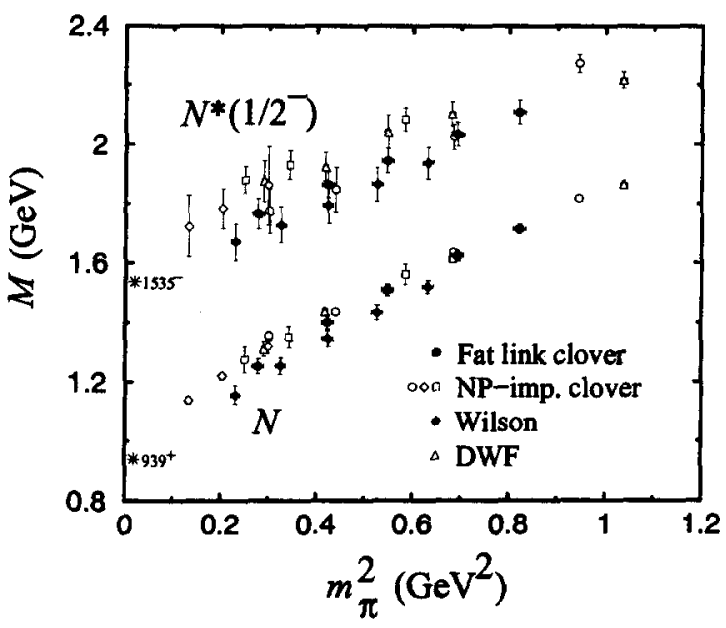

Figure 1. Masses of the nucleon and the lowest $J^{P}=\frac{1}{2}^{-}$excitation. The FLIC and Wilson results are from the present analysis, with the NP improved clover [7] and DWF [6] results shown for comparison. The empirical $N$ and $N^{*}(1535)$ masses are indicated by the asterisks.

results for the nucleon mass lie systematically low in comparison to these due to large $\mathcal{O}(a)$ errors in this action [2]. A similar pattern is repeated for the $N^{*}\left(\frac{1}{2}^{-}\right)$masses. Namely, the FLIC, NP improved clover and DWF masses are in agreement with each other, while the Wilson results again lie systematically low. A mass splitting of around $400 \mathrm{MeV}$ is clearly visible between the $N$ and $N^{*}$ for all actions, including the Wilson action, contrary to previous claims [6].

Fig. 2 shows the ratio of the masses of the $N^{*}\left(\frac{1}{2}^{-}\right)$and the nucleon. Once again, there is good agreement between the FLIC and DWF actions. However, the results for the Wilson action lie above the others, as do those for the anisotropic $\mathrm{D}_{234}$ action [5]. The $\mathrm{D}_{234}$ action has been mean-field improved, and uses an anisotropic lattice which is relatively coarse in the spatial direction $(a \approx 0.24 \mathrm{fm})$. This is an indication of the need for nonperturbative or fat link improvement. 


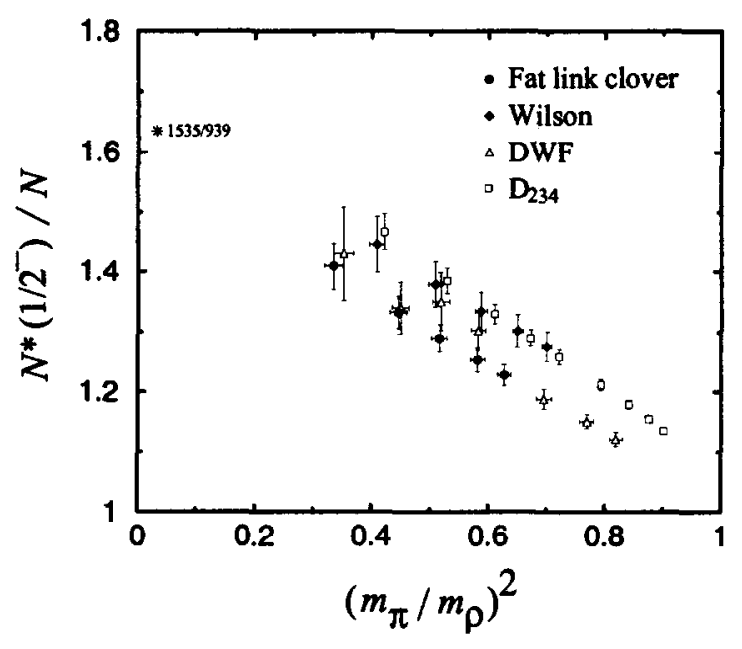

Figure 2. Ratio of the $N^{*}\left(\frac{1}{2}^{-}\right)$and $N$ masses. The FLIC and Wilson results are from the present analysis, with results from the DWF [6] and $D_{234}$ [5] actions shown for comparison. The empirical $N^{*}(1535) / N$ mass ratio is denoted by the asterisk.

The mass splitting between the two lightest nearby $N^{*}\left(\frac{1}{2}^{-}\right)$states $\left(N^{*}(1535)\right.$ and $\left.N^{*}(1650)\right)$ can be studied by considering the $\chi_{1}$ and $\chi_{2}$ interpolating fields in Eqs. (4) and (5). Recall that the "diquarks" in $\chi_{1}$ and $\chi_{2}$ couple differently to spin, so that even though the correlation functions built up from the $\chi_{1}$ and $\chi_{2}$ fields will be made up of a mixture of many excited states, they will have dominant overlap with different states yielding different masses [3]. The results, shown in Fig. 3 for the FLIC action, indicate that indeed the $N^{*}\left(\frac{1}{2}^{-}\right)$corresponding primarily to the $\chi_{2}$ field (labeled " $N_{2}^{*}$ ") lies systematically above the $N^{*}\left(\frac{1}{2}^{-}\right)$associated primarily with the $\chi_{1}$ field (" $N_{1}^{*}$ ").

As has long been known, the positive parity $\chi_{2}$ interpolating field (" $N_{2}$ ", which is also sometimes denoted by " $N^{\prime}\left(\frac{1}{2}^{+}\right)$") does not have good overlap with the nucleon ground state [3], and corresponds to a state which lies around $400 \mathrm{MeV}$ above the negative parity excitation of $\chi_{1}$. There

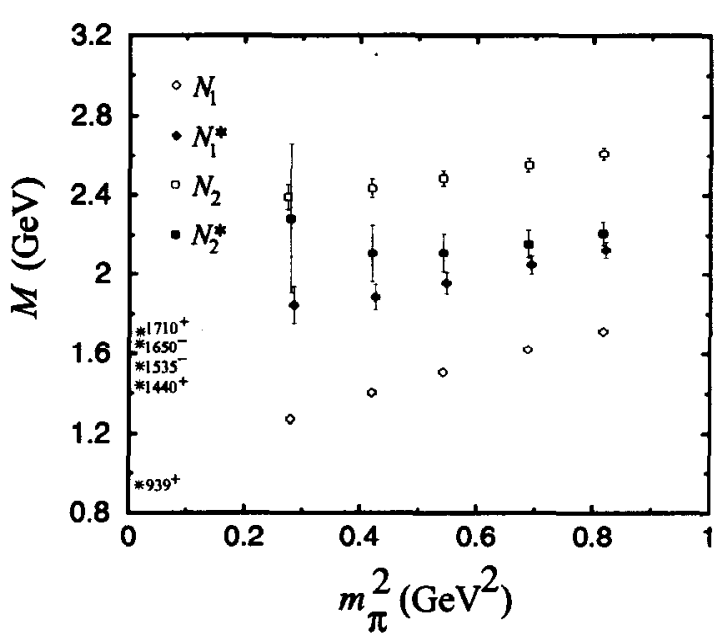

Figure 3. Masses of the $\frac{1}{2}^{+}$and $\frac{1}{2}^{-}$nucleons, for the FLIC action. The positive parity $\left(N_{1}\right.$ and $\left.N_{2}\right)$ and negative parity $\left(N_{1}^{*}\right.$ and $\left.N_{2}^{*}\right)$ states are constructed from the $\chi_{1}^{p}$ and $\chi_{2}^{p}$ interpolating fields, respectively. The empirical masses of lowest two $\frac{1}{2}^{ \pm}$excitations of the nucleon are indicated by the asterisks.

is little evidence that this state is the $N^{*}(1440)$ Roper resonance (first $\frac{1}{2}^{+}$excitation of the nucleon). While it is possible that the Roper resonance may have a strong nonlinear dependence on the quark mass at $m_{\pi}^{2}<0.2 \mathrm{GeV}^{2}$, arising from pion loop corrections, it is unlikely that this behavior would be so dramatically different from that of the $N^{*}(1535)$ so as to reverse the level ordering obtained from the lattice. A more likely explanation is that the $\chi_{2}$ interpolating field does not have good overlap with either the nucleon or the $N^{*}(1440)$, but rather a (combination of) excited $\frac{1}{2}^{+}$state(s).

Recall that in a constituent quark model in a harmonic oscillator basis the mass of the Roper is higher than the mass of the lowest $P$-wave excitation. The lattice data thus appear to be consistent with the naive quark model expectation at large values of $m_{q}$. Better overlap with the Roper resonance is likely to require more exotic interpolating fields. 


\section{CONCLUSION}

We have presented the first results for the excited baryon spectrum from lattice QCD using an $\mathcal{O}\left(a^{2}\right)$ improved gauge action and an improved Fat Link Irrelevant Clover (FLIC) quark action in which only the links of the irrelevant dimension five operators are smeared. The simulations have been performed on a $16^{3} \times 32$ lattice at $\beta=4.60$, providing a lattice spacing of $a=0.125(2) \mathrm{fm}$. The analysis is based on a set of 50 configurations generated on the new Orion computer cluster at the CSSM, Adelaide.

Good agreement is obtained between the FLIC and other improved actions, such as the nonperturbatively improved clover [7] and domain wall fermion [6] actions, for the nucleon and its chiral partner, with a mass splitting of $\sim 400 \mathrm{MeV}$. Our results for the $N^{*}\left(\frac{1}{2}^{-}\right)$improve on those using the $\mathrm{D}_{234}[5]$ and Wilson actions. Despite strong chiral symmetry breaking, the results with the Wilson action are still quite reasonable rendering earlier conjectures invalid. Using the two standard nucleon interpolating fields, we also confirm earlier observations [4] of a mass splitting between the two nearby $\frac{1}{2}^{-}$states. We find no evidence of overlap with the $\frac{1}{2}^{+}$Roper resonance.

We have not attempted to extrapolate the lattice results to the physical region of light quarks, since the nonanalytic behavior of $N^{*}$ 's near the chiral limit is not as well understood yet as that of the nucleon $[10,11]$. It is vital that future lattice $N^{*}$ simulations push closer towards the chiral limit. On a promising note, our simulations with the 4 sweep FLIC action are able to reach relatively low quark masses $\left(m_{q} \sim 60-70 \mathrm{MeV}\right)$ already. We have also not addressed the question of the extent to which quenching may affect any of our results. We naturally expect the effects of quark loops to be relatively unimportant at the currently large quark masses, although quenching may well produce some artifacts as one nears the chiral limit.

For future work, we intend to use variational techniques to better resolve individual excited states, for instance, those corresponding to the $N_{1}^{*}$ and $N_{2}^{*}$ fields (using a $2 \times 2$ correlator ma- trix). In order to further explore the origin of the Roper resonances, more exotic interpolating fields involving higher Fock states, or other nonlocal operators should be investigated. Finally, the present $N^{*}$ mass analysis will be extended in future to include $N \rightarrow N^{*}$ transition form factors through the calculation of three-point correlation functions.

We thank D.G. Richards for providing the data points from Ref.[7]. This work was supported by the Australian Research Council, and the U.S. Department of Energy contract DE-AC05-84ER40150, under which the Southeastern Universities Research Association (SURA) operates the Thomas Jefferson National Accelerator Facility (Jefferson Lab).

\section{REFERENCES}

1. E.A. Veit, B.K. Jennings, R.C. Barrett and A.W. Thomas, Phys. Lett. B 137 (1984) 415; P.B. Siegel and W. Weise, Phys. Rev. C 38 (1988) 2221.

2. J.M. Zanotti et al., hep-lat/0110216; and these proceedings.

3. D.B. Leinweber, Phys. Rev. D 51 (1995) 6383 .

4. F.X. Lee and D.B. Leinweber, Nucl. Phys. B (Proc.Suppl.) 73 (1999) 258.

5. F.X. Lee, Nucl. Phys. B (Proc.Suppl.) 94 (2001) 251.

6. S. Sasaki, T. Blum and S. Ohta, heplat/0102010.

7. D.G. Richards, Nucl. Phys. Proc. Suppl. 94 (2001) 269; M. Göckeler et al., heplat/0106022.

8. D.B.Leinweber, R.W.Woloshyn and T.Draper, Phys. Rev. D 43 (1991) 1659.

9. K. Bowler et al., Nucl. Phys. B240 (1984) 213.

10. D.B. Leinweber, A.W. Thomas, K. Tsushima and S.V. Wright, Phys. Rev. D 61 (2000) 074502.

11. R.D. Young et al., hep-lat/0111041; and these proceedings. 